\title{
Uso de nuevas técnicas y procedimientos endoscópicos en el diagnóstico y seguimiento de la enfermedad celíaca.
}

\author{
Use of new techniques and endoscopic procedures in the diagnosis and \\ monitoring of celiac disease.
}

\author{
Uso de novas técnicas e procedimentos endoscópicos no diagnóstico e \\ monitoramento da doença celíaca.
}

Ximena Rodríguez Olaso. Gastroenteróloga. Ex - Asistente de la Clínica de Gastroenterología.

Carolina Olano Gossweiler Profesora Agregada de la Clínica de Gastroenterología.

Virginia López González Gastroenteróloga. Ex - Profesora Adjunta de la Clínica de Gastroenterología.

Resumen: Desde que inició la toma de biopsias a través de métodos endoscópicos para el diagnóstico de enfermedad celíaca (EC), se ha intentado describir distintos marcadores macr oscópicamente visibles que sugirieran o confirmaran la presencia de atrofia vellositaria en la zona estudiada. Algunos de estos signos son altamente específicos pero su sensibilidad es baja por lo que, por si solos, tienen escaso valor en el algoritmo diagnóstico. El objetivo de esta revisión es analizar la evidencia científica existente sobre la utilidad de las nuevas tecnologías y técnicas endoscópicas en la identificación de marcadores de atrofia vellositaria en el diagnóstico y seguimiento de la enfermedad celíaca Método. Se llevó a cabo una revisión descriptiva de bibliografía donde se buscaron artículos originales en las bases de datos Pubmed y LILACS en el periodo 2006-2016. Resultados. El valor de la cromoendoscopía digital para la búsqueda de marcadores endoscópicos de atrofia vellositaria ha sido escasamente estudiado La sensibilidad de NBI con magnificación se estima entre 83 y 95\%. Los únicos trabajos con $\mathrm{OBI}$ e I-Scan evidenciaron especificidad y sensibilidad perfectas, pero únicamente en pacientes con alta probabilidad de ser celiacos o portadores de atrofia severa. Con respecto a la microscopía endoscópica, la endocitoscopía no parece mejorar el desempeño de la endoscopía de manera significativa, sin embargo, con solo dos trabajos con bajo número de pacientes, la endomicroscopía laser confocal ha mostrado mayor sensibilidad en la detección de marcadores de atrofia. La VCE tiene alta especificidad (95-100\%) pero menor sensibilidad (89\%) y su mayor utilidad se encuentra en objetivar alteraciones que están más allá del alcance de la endoscopía tradicional y valorar su extensión. No existen trabajos que evalúen específicamente su presencia con enteroscopía doble balón, balón único o espiral. Conclusiones. Algunas de las nuevas técnicas y tecnologías parecen ser altamente específicas pero poco sensibles en la detección de marcadores endoscópicos de atrofia vellositaria. Son necesarios más estudios para determinar el valor de técnicas de microscopía endoscópica como herramienta diagnóstica a futuro.

Palabras clave: endoscopía, enfermedad celíaca, atrofia vellositaria, videocapsula endoscópica.

Abstract: Since endoscopic methods are used for biopsying the duodenum at diagnosis or follow-up in celiac disease, attempts have been made to find macroscopically visible markers that suggest or confirm the presence of villous atrophy in the studied area. Some of these signs are highly specific but their sensitivity is low so that, if alone, they have little value in the diagnostic algorithm. The objective of this review is to analyze the existing scientific evidence on the usefulness of new technologies and endoscopic techniques in the identification of markers of villous atrophy in the diagnosis and follow-up of celiac disease. Method. A descriptive review of literature was conducted, where original articles were sought in Pubmed and LILACS databases in the period 2006-2016. Results. The value of digital chromoendoscopy for the search of endoscopic markers of villous atrophy has barely been studied. Sensitivity of NBI with magnification is estimated between 83 and $95 \%$. The only studies with $\mathrm{OBI}$ and I-Scan showed 
perfect specificity and sensitivity, but only in patients with high probability of being celiac or with severe atrophy. Regarding endoscopic microscopy, endocytoscopy does not seem to improve performance in a significant way, however, with only two studies with a small number of patients, confocal laser endomicroscopy has shown greater sensitivity in the detection of atrophy markers. The VCE has high specificity (100\%) but low sensitivity and its greatest utility is finding alterations that are beyond the scope of traditional endoscopy and assess its extent. There are no studies that specifically evaluate the presence of the same signs with double balloon enteroscopy, single balloon or spiral. Conclusions. Some of the new techniques and technologies appear to be highly specific but less sensitive in the detection of endoscopic markers of villous atrophy. More studies are needed to determine the value of endoscopic microscopy techniques as a diagnostic tool in the future.

Keywords: endoscopy, celiac disease, villous atrophy, videocapsule endoscopy.

Resumo: Desde o início das biópsias através de métodos endoscópicos para o diagnóstico de CD, uma tentativa foi feita para descrever diferentes marcadores macroscopicamente visíveis que sugerem ou confirmam a presença de atrofia vilosa na área estudada. Alguns desses sinais são altamente específicos, mas sua sensibilidade é baixa, por si só, eles têm pouco valor no algoritmo de diagnóstico. O objetivo desta revisão é analisar a evidência científica existente sobre a utilidade de novas tecnologias e técnicas endoscópicas na identificação de marcadores de atrofia vilosa no diagnóstico e monitoramento da doença celíaca. Método. Foi realizada uma revisão descritiva da literatura onde os artigos originais foram pesquisados nas bases de dados Pubmed e LILACS no período 2006-2016. Resultados. O valor da cromoendoscopia digital para a busca de marcadores endoscópicos de atrofia vilosa foi mal estudado. A sensibilidade do $\mathrm{NBI}$ com ampliação é estimada entre 83 e 95\%. O único trabalho com OBI e I-Scan mostrou especificidade e sensibilidade perfeita, mas apenas em pacientes com alta probabilidade de serem celíacos ou portadores de atrofia grave. Em relação à microscopia endoscópica, a endocitoscopia não parece melhorar o desempenho da endoscopia de forma significativa, no entanto, com apenas dois estudos com um número reduzido de pacientes, a endomicroscopia confocal laser mostrou maior sensibilidade na detecção de marcadores de atrofia. O VCE tem alta especificidade (95-100\%), mas menor sensibilidade (89\%) e sua maior utilidade é objetivar alterações que estão além do alcance da endoscopia tradicional e avaliam sua extensão. Não há estudos que avaliem especificamente sua presença com balão duplo, bola única ou enteroscopia espiral. Conclusões. Algumas das novas técnicas e tecnologias parecem ser altamente específicas, mas não muito sensíveis na detecção de marcadores endoscópicos de atrofia vilosa. Mais estudos são necessários para determinar o valor das técnicas de microscopia endoscópica como ferramenta de diagnóstico no futuro.

Palavras-chave: endoscopia, doença celíaca, atrofia vilosa, cápsula de vídeo endoscópico.

Recibido: 16/09/2017 - Aceptado: 08/02/2018

Clínica de Gastroenterología - Prof. Dr. Henry Cohen. Hospital de Clínicas. Facultad de Medicina. Universidad de la

República. Montevideo, Uruguay.

Correspondencia: E-mail: xrodriguezo@gmail.com 


\section{Introducción}

La enfermedad celíaca (EC) es una patología sistémica en la que individuos genéticamente susceptibles expuestos al gluten desarrollan una injuria autoinmune principalmente dirigida al intestino ${ }^{(1)}$.

La enteropatía resultante es la causa más frecuente de alteraciones histopatológicas del intestino delgado en el hemisferio occidental. En 1990 Marsh describió la sucesión de cambios histológicos que se dan en la mucosa duodenal del paciente con enfermedad celíaca ${ }^{(2)}$. Estos cambios a nivel histológico relacionados con la ingesta de gluten son necesarios para el diagnóstico de EC y la biopsia intestinal sigue siendo recomendada en el adulto para confirmar su presencia ${ }^{(3)}$.

Desde que se inició la toma de biopsias a través de métodos endoscópicos se ha intentado usar distintos marcadores macroscópicamente visibles que sugieran o confirmen la presencia de atrofia en la zona estudiada. Para ello se describieron varios signos: la disminución en número y altura de los pliegues, el patrón en mosaico y el signo del peine a nivel duodenal y la visualización de los vasos por transparencia y la nodularidad del bulbo duodenal como elementos relacionados con atrofia vellositaria histológica ${ }^{(4,5)}$ Figura 1.

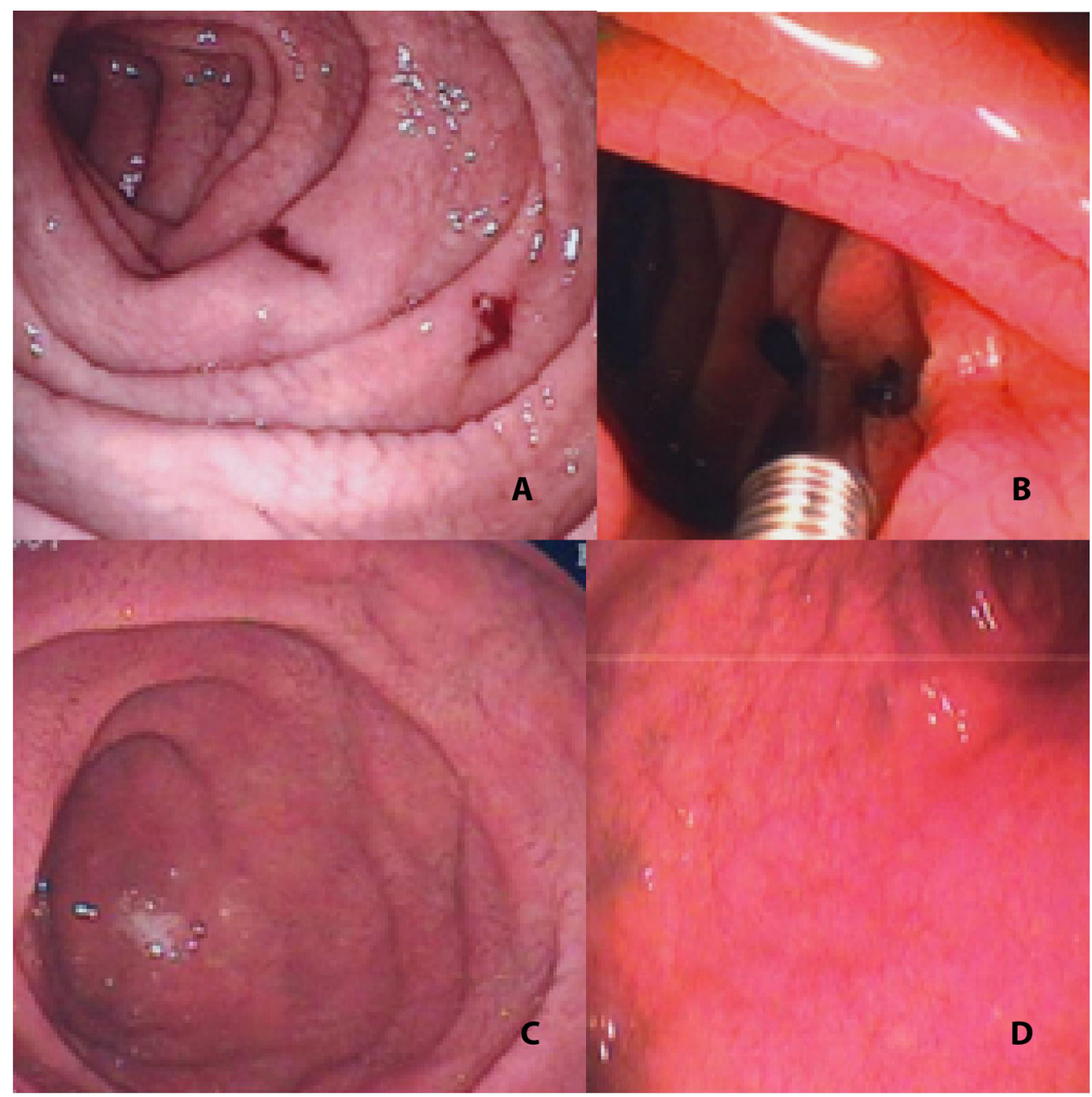

Algunos de estos son altamente específicos pero su sensibilidad es baja por lo que disminuye su valor en el algoritmo diagnóstico.

En los últimos años, con el desarrollo de nuevos abordajes endoscópicos y nuevas técnicas para optimizar la imagen se ha cuestionado nuevamente el lugar de la endoscopía como elemento diagnóstico único o como guía para la toma de biopsias y la optimización de recursos. 
El objetivo de esta revisión es analizar la evidencia científica existente sobre la utilidad de las nueva tecnologías y técnicas endoscópicas en la identificación de marcadores de atrofia vellositaria (MEAV) en el diagnóstico y seguimiento de la EC.

Con este fin, se llevó a cabo una revisión descriptiva de bibliografía donde se revisaron las bases de datos Pubmed y LILACS en el periodo 2006-2016 y como palabras claves se usaron: endoscopía, cromoendoscopía, endomicroscopía, microscopía láser confocal, videocápsula endoscópica, enteroscopía y atrofia vellositaria. Los criterios de selección de los artículos científicos hallados durante la búsqueda fueron: artículos originales publicados en inglés, español o portugués. También se realizó una investigación de las referencias utilizadas por las publicaciones más relevantes

\section{Resultados}

Para un mejor análisis de los avances de la endoscopía digestiva en la última década se realizó una categorización en dos grupos: tecnologías actuales aplicadas a la video-endoscopía y nuevas técnicas endoscópicas disponibles.

\section{A) Tecnologías actuales aplicadas a la video-endoscopía}

Los procedimientos endoscópicos con luz blanca estándar permiten la identificación de varios MEAV: disminución de número y altura del los pliegues, indentación de los mismos (signo del peine), patrón micronodular de la mucosa, patrón en mosaico y visualización de vasos por transparencia ${ }^{(4-6)}$. La sensibilidad y especificidad de estos marcadores se encuentra en el rango del 6 a $94 \%$ y de 83 a 100\% respectivamente ${ }^{(7-10)}$. La gran variabilidad de la sensibilidad puede estar relacionada con la ausencia de estos marcadores en la enfermedad temprana y la presencia de alteraciones parcheadas del intestino delgado, así como también con diferencias entre los endoscopios usados y la experiencia de los endoscopistas participantes en cada estudio.

Dado que la eficacia diagnóstica de la endoscopía convencional para la búsqueda de atrofia vellositaria es baja, es obligatorio realizar toma de biopsias de bulbo duodenal y segunda porción de duodeno en número significativo (2 y 4 respectivamente) al azar para descartar daño histológico ${ }^{(11)}$. En los intentos por mejorar esta capacidad de diagnóstico se han desarrollado diferentes tecnologías dirigidas a mejorar la visualización de la mucosa duodenal.

\section{A.1) Cromoendoscopía digital}

A diferencia de los endoscopios de fibra óptica que se usaban anteriormente, los videoendoscopios obtienen imágenes como señales electrónicas que pueden ser analizadas usando varias técnicas de procesamiento. El mejoramiento en la imagen es una técnica comúnmente usada para mejorar la capacidad diagnóstica.

La cromoendoscopía digital (CED) eliminaría la necesidad de métodos directos de tinción con colorantes vitales y las dificultades que esto conlleva en la práctica clínica diaria (mayor tiempo de endoscopía/ anestesia, mayor requerimiento de materiales, etc) ${ }^{(12)}$.

La imagen endoscópica con CED puede lograrse por uso de filtros en la fuente de luz del endoscopio como se hace con el Narrow Band Imaging (NBI) desarrollado por Olympus o por procesamiento posterior de la imagen obtenida por reconstrucción digital. En esta última modalidad se han desarrollado dos tecnologías: el Optimal Band Imaging (OBI) y el I-Scan desarrollados por Fuji y Pentax respectivamente. (Figura 2 y Figura 3) 
Figura 2: Imágenes de DII obtenidas por videoendoscopía digestiva alta con CED (OBI)-

Figura 3: imágenes de DII obtenidas por videoendoscopía digestiva alta con CED $(\mathrm{OBI})$ - ensanchamiento focal de algunas vellosidades (con magnificación)
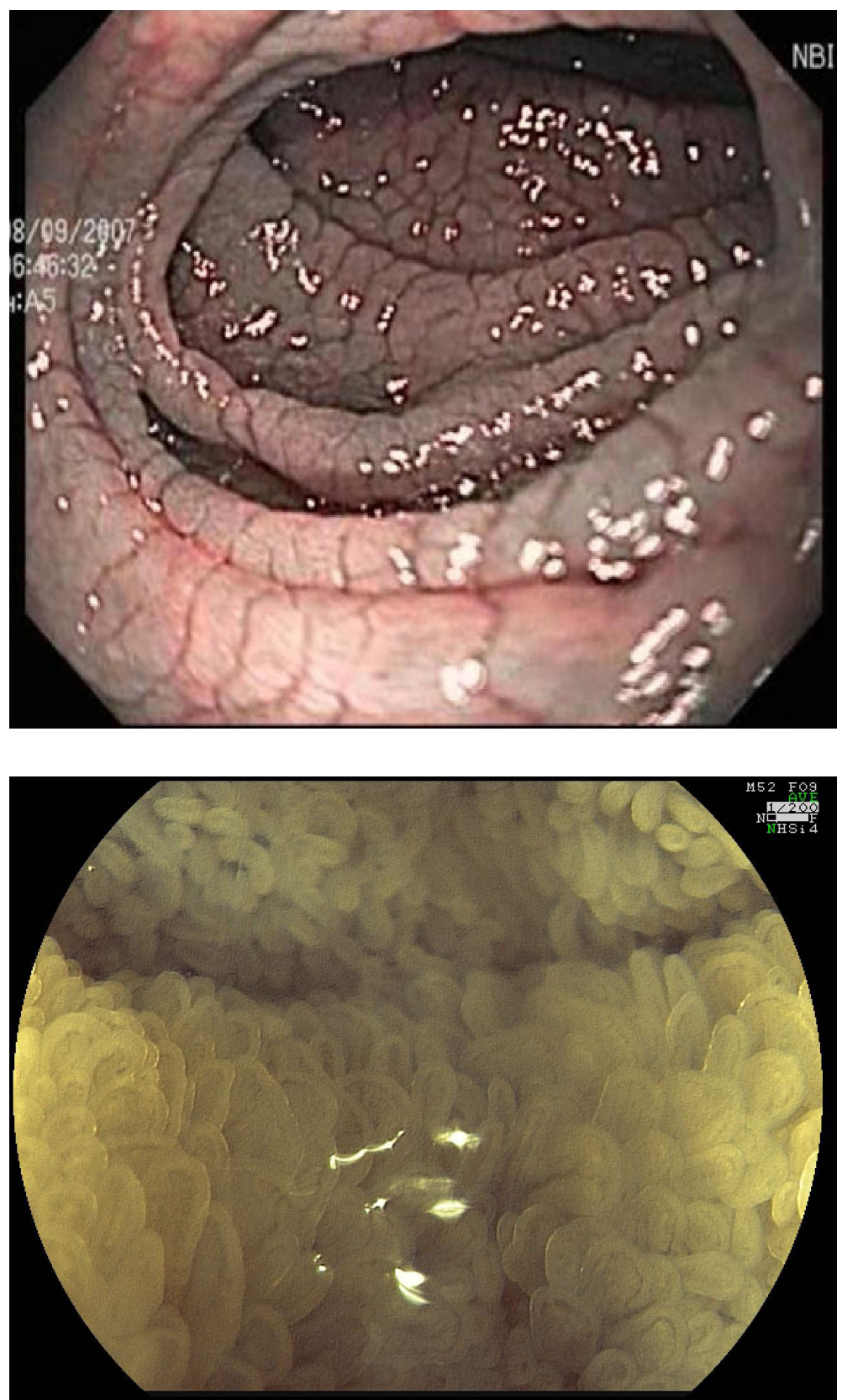

Narrow Band Imaging (NBI): El principio físico fundamental subyacente es que la profundidad de penetración de la luz en los tejidos es proporcional a la longitud de onda utilizada: luz azul para el epitelio superficial, verde para el intermedio y rojo para el tejido profundo. La luz blanca penetra solo superficialmente y es absorbida por la hemoglobina. Todos los endoscopios con NBI permiten cambiar de filtro con un sencillo movimiento de los controles para observar lo mismo con luz blanca, filtro azul o verde, logrando un mejor contraste de la mucosa y los vasos (12). En general este procedimiento se realiza asociado a uno de magnificación, por inmersión o digital para mejorar la definición de lesiones estructurales.

A priori esto aumentaría considerablemente nuestra capacidad de valorar MEAV y lograría idealmente evitar la necesidad de biopsias o alternativamente guiaría a lugares específicos donde suponemos el rendimiento diagnóstico sería mayor que si se realizaran al azar.

En todos los trabajos valorados, el NBI se asoció con algún tipo de magnificación (por inmersión en agua, magnificación óptica o digital). En todos los casos la evaluación de los resultados fue comparado con los histológicos que son el patrón de referencia. La sensibilidad del NBI/magnificación para valorar MEAV se estimó entre 83 y 95\% ${ }^{(13-15)}$.

El valor predictivo positivo fue en todos los casos del 100\%, por lo que se concluyó que el $\mathrm{NBI}$ era un método relativamente fácil de incorporar que podría guiar la toma de biopsias hacia 
zonas con mucosa alterada. Si la atrofia fuera visualizada por NBI, podría disminuir el número de biopsias necesarias, inclusive en niños, se ha postulado podría ser una sola muestra histológica según Valitutti y otros ${ }^{\left({ }^{14}\right)}$. Sin embargo, frente a la ausencia de MEAV debe continuarse con el protocolo estándar de toma de biopsias de bulbo y D II (2 y 4 respectivamente).

Optimal Band Imaging (OBI): Solo se encontró un trabajo original que valoró el uso de esta técnica para buscar MEAV ${ }^{(16)}$, que se realizó con OBI y magnificación. Este estudio mostró valores perfectos de sensibilidad y especificidad, sin embargo incluyó un escaso número de pacientes $(\mathrm{N}=31)$ y todos con alta probabilidad pretest de tener $\mathrm{EC}$, por lo que estos datos no son extrapolables a otras poblaciones. Son necesarios más estudios para confirmar estos datos.

I-Scan: El único trabajo original se realizó en pacientes con sospecha clínica o serológica de enfermedad celiaca u otra patología malabsortiva. Los MEAV fueron comparados con la histología convencional obtenida en el mismo momento. El estudio se realizó en 115 pacientes y demostró que cuando la atrofia histológica era total la concordancia con el I-Scan era del $100 \%$ (sensibilidad y especificidad de 100\%). Sin embargo, en los patrones con atrofia parcial y mucosa normal, los resultados fueron menos exactos con sensibilidades de 77 y $94 \%$ y especificidades de 94 y $84 \%$.

Por lo antes mencionado, se concluye que este tipo de CED podría ser útil para guiar la toma de biopsias, pero su uso por ahora no sustituiría la toma de tejido para histología convencional. Un dato importante es que a diferencia de otros trabajos realizados con NBI y OBI, en este se decidió por protocolo no usar magnificación, por lo que estos datos podrían cambiar en trabajos posteriores que usen ambas ${ }^{(17)}$.

\section{A.2) Microscopía endoscópica}

El desarrollo de técnicas de imagen de endomicroscopía permite la valoración cito e histológica in vivo con todas las ventajas que ésta puede suponer (menos tiempo al diagnóstico, valoración del tejido y las células en su estado natural, etc.)

Algunos de estos procedimientos se encuentran ya disponibles para uso clínico y son los que formaron parte de la búsqueda en esta revisión, mientras que otros están aún en fase experimental (endoscopía multifotón, imagenología molecular por endoscopía).

Endocitoscopía: Permite la valoración de la mucosa superficial por microscopía de contacto con luz blanca. Esta técnica usa lentes objetivo de alto poder y requiere mantener la superficie fija para hacer foco en la zona a estudiar. En general se administra previamente algún agente mucolítico y se requieren colorantes tópicos (Ej. cristal violeta, azul de metileno) ${ }^{(18)}$.

Basados en la elevada magnificación lograda con esta técnica se esperaba una excelente visualización a nivel celular y mayor habilidad para detectar los cambios tempranos de la EC (aumento de LIES, ausencia de borde en cepillo, etc.). Sin embargo, ninguno de los 2 trabajos evaluados pudo mejorar el desempeño de la endoscopía de manera significativa ${ }^{(19,20)}$. Tanto en la evaluación con magnificación 450x como con 1100x, los únicos elementos con alta especificidad fueron la disminución del número de vellosidades y la hiperplasia críptica ${ }^{(19)}$, los mismos que pueden ser valorados por otros métodos ya referidos.

Endoscopía Láser Confocal (ELC): Se puede realizar por 2 métodos: colocación de un microscopio láser confocal en el extremo distal del endoscopio (Pentax, EEUU, discontinuado) o mediante la utilización de una pequeña sonda introducida por el canal operador del endoscopio (Cellvizio, Francia). Durante el procedimiento se "ilumina" la mucosa con láser, lo que permite la adquisición de imágenes histológicas in-vivo con una magnificación 1000x. Requiere el uso de colorantes fluorescentes tópicos o sistémicos ${ }^{(21)}$.

Se evaluaron 2 trabajos publicados, uno con cada uno de los métodos antes mencionados.

En el primero, realizado en el año 2008, los autores definieron un sistema de puntuación (número de imágenes con atrofia vellositaria y/o hiperplasia críptica/número de total de imágenes adquiridas en el mismo paciente). Con más de 6 imágenes en 100 (puntuación: 0,06) la exactitud diagnóstica fue de $95 \%$ (sensibilidad 94\% y especificidad 92\%) ${ }^{(22)}$.

Pohl y cols. realizaron en el año 2011 un trabajo que testea el uso de esta tecnología en la evaluación de los MEAV ${ }^{(23)}$. El primer objetivo fue identificar los elementos encontrados por ELC que mejor predicen la presencia de patología duodenal para luego valorar su eficacia en una muestra de biopsias aleatorizadas. Es así que se consideraron MEAV clásicos (disminución de la altura y número de las vellosidades, irregularidad de las mismas, hiperplasia críptica) y también algunos nuevos cuya observación podría realizarse al lograr mayor magnificación (aumento de 
Ios LIES, densidad celular en el subepitelio, valoración vascular: regularidad y morfología de los vasos). De todos estos elementos, los únicos con valor predictivo para EC fueron la atrofia vellositaria y la irregularidad en la morfología de las vellosidades, el resto o bien no pudo ser evaluado en números significativos (LIES y densidad celular) o no tuvo valores aceptables de sensibilidad y/o especificidad.

\section{B) Nuevas técnicas endoscópicas}

\section{B.1) Video Cápsula Endoscópica (VCE)}

Es un dispositivo de reducidas dimensiones que, a través de la ingestión oral, permite la obtención de imágenes del tubo digestivo durante su recorrido fisiológico a través de éste. Es un estudio mínimamente invasivo, bien tolerado.

Existen distintos tipos de cápsulas para valorar diferentes sectores (esófago, intestino delgado (ID), colon). En esta revisión solo hablaremos de las destinadas a valorar el ID que por sus características son un método de evaluación que puede ser útil en pacientes celiacos o con sospecha de EC (Figura 4 y Figura 5).

Figura 4: Imagen obtenidas con VCE Pillcam SB2 de una paciente con enfermedad

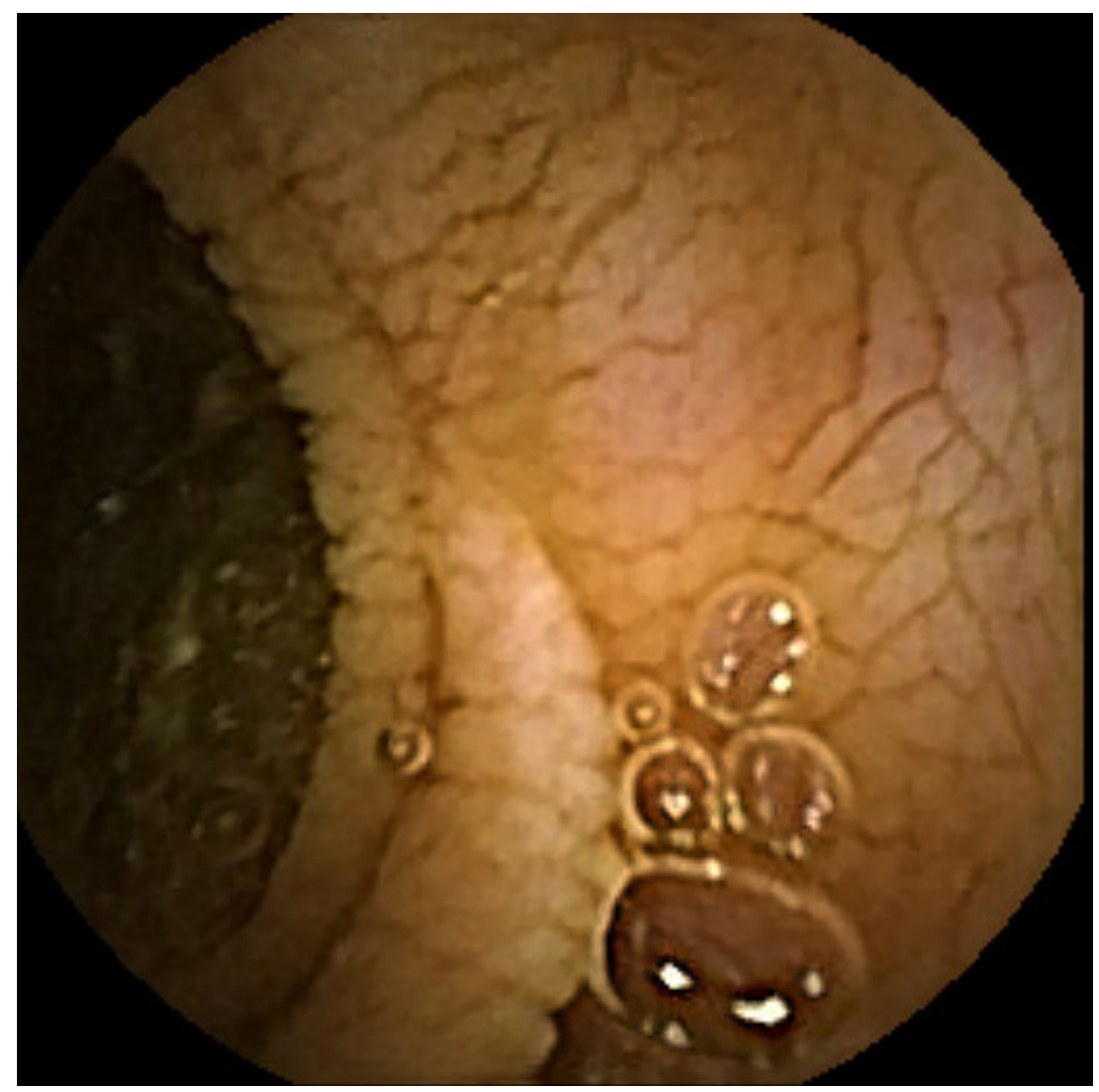




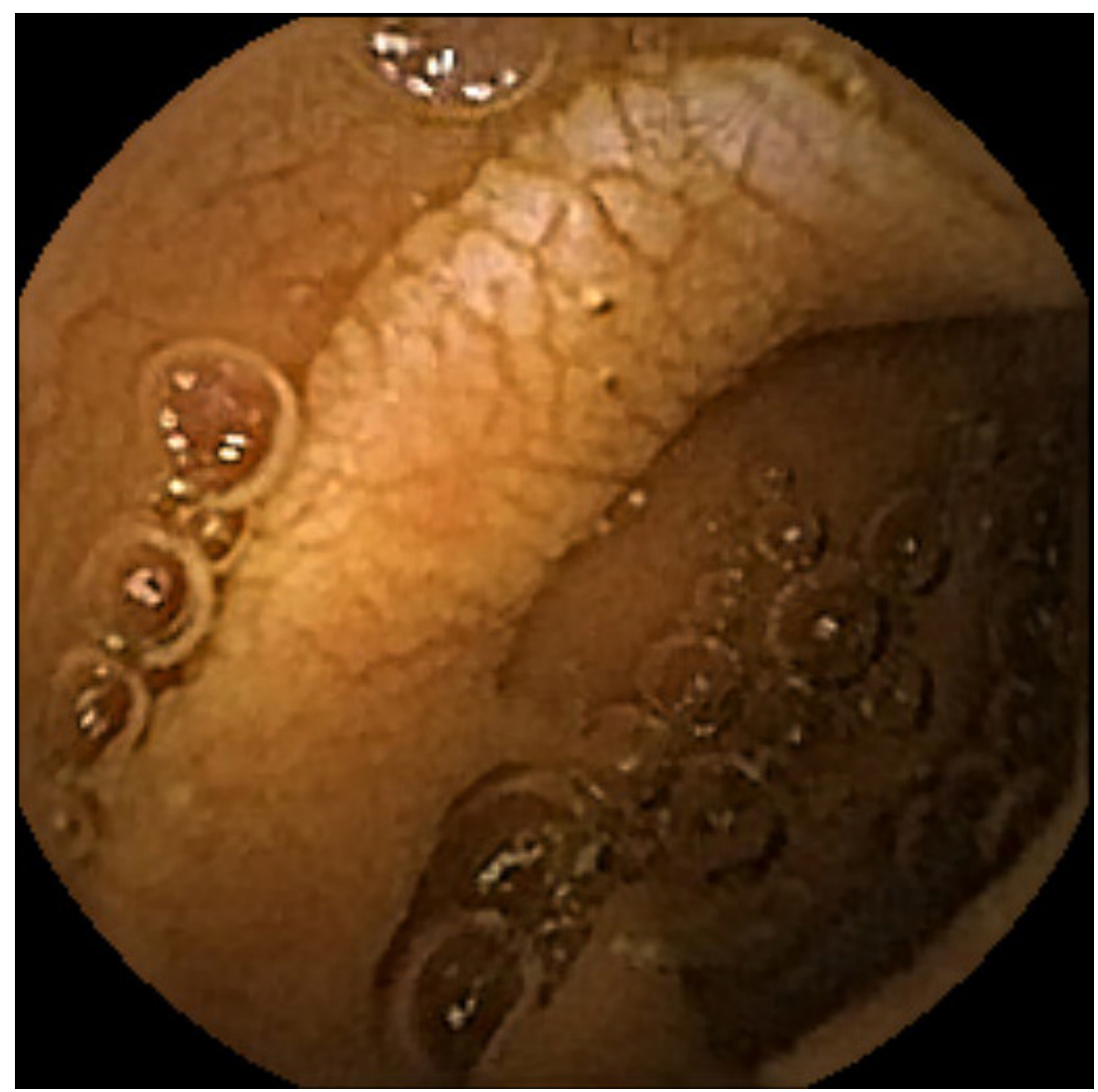

Hasta el momento se dispone en el mercado de 6 modelos de VCE desarrolladas por igual número de empresas: Pillcam, Endocapsule, Mirocam, OMOM, Navicam y Capsocam (24). La original y más usada, Ilamada Pillcam SB, fue desarrollada por Given Imaging (Israel). Salió a la venta en el año 2000 y desde entonces han aparecido sucesivos modelos con mejoras adicionales (mayor ángulo de visión, mayor definición, etc). Todos los trabajos que cumplieron los criterios de búsqueda fueron realizados con Pillcam M2A.

Los estudios que evaluaron la cápsula en el diagnóstico de EC comparan el hallazgo de MEAV con la presencia o ausencia de atrofia confirmada histológicamente.

El primer trabajo prospectivo y ciego que evaluó el desempeño de la VCE para el diagnóstico de atrofia vellositaria fue realizado en el año 2005 por Petronienne y cols. La sensibilidad fue $70 \%$ con especificidad de $100 \%$. Aún cuando fue un estudio con pocos casos $(\mathrm{N}=10)$ demostró ya la importancia de la experiencia del observador en los resultados obtenidos ${ }^{(25)}$.

En años sucesivos se realizaron varios estudios, el más numeroso, multicéntrico, realizado por Rondonotti y cols. ${ }^{(26)}$ obtuvo una sensibilidad y especificidad de 88 y $91 \%$ respectivamente. En el metanálisis del año 2012 de Rokkas y Niv, la sensibilidad agrupada fue de $89 \%$ y la especificidad de $95 \%{ }^{(27)}$. Es importante considerar que la técnica de referencia (VEDA con biopsias) es más barata y accesible en general por lo que en todos los casos se concluye que la misma sigue siendo el patrón de referencia. Otro aporte de la VCE al diagnóstico de la EC podría estar en valorar su extensión, no existiendo acuerdo si ésta tiene relación con la severidad de los síntomas ${ }^{(25,28)}$. Se propone que ésta sería una línea de investigación para el futuro.

En los últimos años se comenzó a usar la CED (FICE y Blue Mode) en la VCE. Hasta la fecha no existen trabajos que valoren su utilidad para el diagnóstico de MEAV.

\section{B.2) Enteroscopía}

Es el estudio endoscópico del intestino delgado $\left(29^{-311}\right.$. Clásicamente se realizaba a través de una ostomía quirúrgica (enteroscopía intraoperatoria) o por abordaje oral o colorrectal por pulsión. Desde el año 2001 en que Yamamoto creó el enteroscopio con doble balón ${ }^{(32)}$ se desarrollaron 2 nuevos instrumentos más: el enteroscopio con balón único y el enteroscopio en espiral que mejoran la canulación del intestino delgado y disminuyen algunas de las complicaciones del procedimiento. 
Debido a que éste es un estudio endoscópico invasivo habitualmente más prolongado, que requiere sedación para su realización y con un índice de complicaciones levemente superior, a priori debería quedar reservado para pacientes con indicación precisa.

Los MEAV que se valoran con esta técnica son los ya descritos para la VEDA.

Enteroscopía de doble balón (EDB): Desarrollada por Fujinon Inc. (Japón). Consiste en un endoscopio de $200 \mathrm{cms}$ y un sobretubo flexible de $140 \mathrm{cms}$, ambos con un balón de látex inflable en su extremo distal. Mediante insuflación y desinsuflación coordinada de los balones se logra avanzar a través del ID.

La mayoría de los trabajos sobre enteroscopía valorados han sido realizados con esta técnica que es la que más tiempo lleva en el mercado.

No existen trabajos que valoren específicamente la presencia de MEAV con esta técnica.

La EDB puede ser útil en pacientes con malabsorción de origen incierto con evaluación previa por VGC y VCC normal. Así lo sugiere un trabajo de Fry y cols. En el que se realizó un diagnóstico etiológico no encontrado previamente en un tercio de los pacientes, entre ellos el de EC ${ }^{(33)}$

Es de destacar que la utilización de OBI como CED no mejora la detección de MEAV (34).

En series que valoran específicamente su utilidad en pacientes con sospecha de EC complicada se observó que es un estudio útil que modifica el manejo terapéutico en $33 \%$ de los pacientes ${ }^{(35)}$. Cabe señalar que tanto los hallazgos positivos como los negativos provocaron cambios terapéuticos. El hallazgo de neoplasias determinó la realización de cirugía, con buena evolución en la serie mencionada, y el descartar monoclonalidad en las biopsias de los enfermos con EC refractaria motivó el inicio de tratamientos médicos inmunosupresores.

Enteroscopía con balón único (EBU): Desarrollada por Olympus Inc. (EEUU). Es un sistema que tiene un solo balón localizado en el sobretubo. Para lograr el avance del endoscopio se usa una combinación de succión y angulación del extremo distal del endoscopio.

El único trabajo con esta técnica aprovecha la ventaja de la enteroscopía para valorar duodeno distal (D IV) y yeyuno y describe pacientes celíacos con MEAV y lesiones histológicas no accesibles a la VEDA convencional. Este trabajo de Kav y col. ${ }^{\left({ }^{36}\right)}$, prospectivo, encuentra que todos los pacientes con EC tuvieron MEAV en algún sector duodeno-yeyunal pero que en 4,5\% solo había compromiso distal. Estos últimos no serían diagnosticados por VEDA convencional y quedarían como celíacos potenciales o sin diagnóstico. Los investigadores en este caso plantean además, el uso para la valoración futura de pacientes de una clasificación según localización endoscópica de los MEAV encontrados por enteroscopía. En este mismo estudio se concluye que la utilización de NBI como CED aplicada a la EBU no mejora el rendimiento diagnóstico con respecto al hallazgo de MEAV.

Enteroscopía en espiral: Se logra con la adaptación de un sobretubo llamado Discovery SB desarrollado por Spirus Medical Inc. (EEUU) a cualquier endoscopio estándar. El mecanismo por el que se avanza es similar al funcionamiento de un sacacorchos.

Es el método más nuevo de los mencionados y todavía no hay trabajos originales que evalúen su uso en valoración de MEAV.

\section{Conclusiones}

Con la introducción de nuevas herramientas endoscópicas de diagnóstico se ha intentado guiar o sustituir la toma de material para histología en el diagnóstico y seguimiento de la EC.

El valor de la cromoendoscopía digital para la búsqueda de marcadores endoscópicos de atrofia vellositaria ha sido escasamente estudiado La sensibilidad de NBI con magnificación se estima entre 83 y 95\%. Los únicos trabajos con OBI e I-Scan evidenciaron especificidad y sensibilidad perfectas, pero únicamente en pacientes con alta probabilidad de ser celíacos o portadores de atrofia severa.

La microscopía endoscópica es una tecnología de reciente uso en la práctica clínica para la valoración a nivel celular e histológico en vivo. La endomicroscopía láser confocal, todavía en etapa experimental, promete alto rendimiento pero se necesitan aún trabajos clínicos de buen diseño que evalúen su sensibilidad y especificidad. 
La VCE tiene alta especificidad (100\%) pero baja sensibilidad y su mayor utilidad reside en objetivar alteraciones más allá del alcance de la endoscopía tradicional y valorar su extensión. No existen trabajos que evalúen específicamente la presencia de los mismos con enteroscopía doble balón, balón único o espiral.

De lo antes expuesto se desprende que ha sido posible mejorar la visión de la mucosa intestinal en cuanto a definición y extensión pero todavía es necesario optimizar estos métodos para lograr sustituir la histología convencional en el diagnóstico de EC. 


\section{Bibliografía}

1- Rewers M. Epidemiology of celiac |disease: what are the prevalence, incidence and progression of celiac disease? Gastroenterology. 2005; 128: s47-s51.

2- Marsh MN. Grains of truth: evolutionary changes in small intestinal mucosa in response to environmental antigen challenge. Gut. 1990; 31:111-114.

3- Ludvigsson J, Leffler D, Bai J, Biagi F, Fasano A, Green P, et al. The Oslo definitions for celiac disease and related terms. Gut 2013; 62(1):43-52.

4- Lee S, Green P. Endoscopy in celiac disease. Curr Opin Gastroenterol. 2005;21:589-594.

5- Cammarota G, Fedeli P, Gasbarrini A. Emerging technologies in upper gastrointestinal endoscopy and celiac disease. Nat Clin Pract Gastroenterol Hepatol. 2009;6:47-56.

6- Brocchi E, Tomassetti P, Misitano B, Epifanio G, Corinaldesi R, Bonvicini F, et al. Endoscopic markers in adult celiac disease. Dig Liver Dis. 2002;34(3):177-82.

7- Bardella M, Minoli G, Radaelli F, Quatrini M, Bianchi P, Conte D. Reevaluation of duodenal endoscopic markers in the diagnosis of celiac disease. Gastrointest Endosc. 2000;51:714-6.

8- Lecleire S, Di Fiore F, Antonietti M, Savoye G, Lemoine F, Le Pessot F, et al. Endoscopic markers of villous atrophy are not useful for the detection of celiac disease in patients with dyspeptic symptoms. Endoscopy. 2006;38:696-701

9- Oxentenko A, Grisolano S, Murray J, Burgart L, Dierkhising R, Alexander J. The sensitivity of endoscopic markers in celiac disease. Am J Gastroenterol. 2002;97:933-8.

10- Mauriño E, Bai J. Endoscopic markers of celiac disease. Am J Gastroenterol. 2002;97:760-1.

11- Cammarota G, Fedeli P, Gasbarrini A. Emerging technologies in upper gastrointestinal endoscopy and celiac disease. Nat Clin Pract Gastroenterol Hepatol. 2009;6:47-56.

12- Jang JY. The past, present and future of image-enhaced endoscopy. Clin Endosc. 2015;48:466-75.

13- Singh R, Nind G, Tucker G, Nguyen N, Holloway R, Bate J, et al. Narrow-band imaging in the evaluation of villous morphology: a feasibility study assessing a simplified classification and observer agreement. Endoscopy. 2010;42:889-94.

14- Valitutti F, Oliva S, Iorfida D, Aloi M, Gatti S, Trovato CM, et al. Narrow band imaging combined with water immersion technique in the diagnosis of celiac disease. Dig Liver Dis. 2014;46:1099-102.

15- Goswami A, Dadhich S, Bhargava N. Use of narrow band imaging in assessing duodenal villous atrophy. Indian J Gastroenterol. 2014;33:440-4.

16- Cammarota G, Cesaro P, Cazzato A, Fedeli P, Sparano L, Vecchio F, et al. Optimal band imaging system: a new tool for enhacing the duodenal villous pattern in celiac disease. Gastrointest Endosc. 2008;68:352-7.

17- Cammarota G, Ianiro G, Sparano L, La Mura R, Ricci R, Larocca L, et al. Image-Enhaced Endoscopy with I-scan Technology for the evaluation of duodenal villous patterns. Dig Dis Sci. 2013;58:1287-92.

18- Kwon RS, Wong Kee Song LM, Adler DG, Conway JD, Diehl DL, Farraye FA, et al. Endocytoscopy. Gastrointest Endosc. 2009;70:610-3.

19- Pohl H, Rösch T, Tanczos BT, Rudolph B,Schlüns K, Baumgart DC. Endocytoscopy for the detection of microestructural features in adult patients with celiac sprue: a prospective, blinded endocytoscopyconventional histology correlation study. Gastrointest Endosc. 2009,70:933-41.

20- Matysiak-Budkin T, Coron E, Mosnier JF, Le Rhun M, Inoue H, Galmiche JP. In vivo real-time imaging of human duodenal mucosal structures in celiac disease using endocytoscopy. Endoscopy. 2010;42:191-

21- Knieling F, Waldner MJ. Light and sound - emerging imaging techniques for inflammatory bowel disease. World J Gastroenterol. 2016 Jul 7;22(25):5642-54.

22- Leong RW, Nguyen NQ, Meredith CG, Al-Sohaily S, Kukic D, Delaney PM, et al. In vivo confocal endomicroscopy in the diagnosis and evaluation of celiac disease. Gastroenterology. 2008;135:1870-6.

23- Pohl H, Tanczos B, Rudolph B, Meining A, Khalifa A, Rösch T, et al. Probe-based confocal laser microscopy identifies criteria predictive of active celiac sprue. Dig Dis Sci. 2012;57:451-7. 
24- Li Z, Carter D, Eliakim R, Wenbiz Z, Wu H, Liao Z,et al. The current main types of capsule endoscopy. En: Li Z, Liao Z, MacAlindon M. Handbook of capsule endoscopy. Berlin: Springer, 2014.

25- Petroniene R, Dubcenco C, Baker J, Ottaway C, Tang SJ, Zanati S, et al. Given $₫$ Capsule endoscopy in celiac disease: evaluation of diagnostic accuracy and interobserver agreement. Am J Gastroeterol. 2005;100:685-94.

26- Rondonotti E, Spada C, Cave D, Pennazio M, Riccioni ME, De Vitis I, et al. Video capsule enteroscopy in the diagnosis of celiac disease: a multicenter study. Am J Gastroenterol. 2007;102:1624-1631.

27- Rokkas T, Niv Y. The role of video capsule endoscopy in the diagnosis of celiac disease: a metaanalysis. Eur J Gastroeterol Hepatol. 2012;24:303-8.

28- Lidums I, Cummins AG, Teo E. The role of capsule endoscopy in suspected celiac disease patients with positive celiac serology. Dig DIs Sci. 2011;56:499-505.

29- Kurien M, Evans KE, Aziz I, Sidhu R, Drew K, Rogers TL, et al. Capsule endoscopy in adult celiac disease: apotential role in equivocal cases of celiac disease?. Gastrointest Endosc. 2013;77:227-32.

30- Maiden L, Elliott T, McLaughlin SD, Ciclitira P. A blinded pilot comparison of capsule endoscopy and small bowel histology in unresponsive celiac disease. Dig Dis Sci. 2009;54:1280-83.

31- Atlas D, Rubio-Tapia A, Van Dyke C, Lahr B, Murray J. Capsule endoscopy in non responsive celiac disease. Gastrointest Endosc. 2011;71:1315-22.

32- Yamamoto H, Sekine Y, Sato Y, Higashizawa T, Miyata T, lino S, et al. Total enteroscopy with a nonsurgical steerable double-ballon method. Gastrointest Endosc. 2001;53:216-20.

33- Fry LC, Belluti M, Neumann H, Malfertheiner P, Monkemuller K. Utility of double-balloon enteroscopy for the evaluation of malabsorption. Dig Dis. 2008;26:134-9.

34- Neumann H, Fry LC, Bellutti M, Malfertheiner P, Monkemuller K. Double-balloon enteroscopy-assisted virtual chromoendoscopy for small-bowel disorders: a case series. Endoscopy. 2009;41:468-71.

35- Tomba C, Sidhu R, Sanders DS, Mooney PD, Branchi F, Locatelli M, et al. Celiac disease and doubleballoon enteroscopy: What can we achieve?: The experience of 2 european tertiary referral centers. J Clin Gastroenterol. 2016;50:313-7.

36- Kav T, Sokmensuer C, Sivri B. Enteroscopic findings of celiac disease and their correlation with mucosal histopathologic changes. Comput Biol Med. 2015;65:315-9.

Pie de fotografías del artículo 